\title{
Principle of Duality on Prognostics
}

\author{
Mohammad Samie \\ School of Applied Sciences \\ Cranfield University \\ Bedford, UK
}

\author{
Amir M. S. Motlagh \\ Faculty of Science and Technology \\ University of Westminster \\ London, UK
}

\author{
Alireza Alghassi \\ School of Applied Sciences \\ Cranfield University \\ Bedford, UK
}

\author{
Suresh Perinpanayagam \\ School of Applied Sciences \\ Cranfield University \\ Bedford, UK
}

\author{
Epaminondas Kapetanios \\ Faculty of Science and Technology \\ University of Westminster \\ London, UK
}

\begin{abstract}
The accurate estimation of the remaining useful life (RUL) of various components and devices used in complex systems, e.g., airplanes remain to be addressed by scientists and engineers. Currently, there area wide range of innovative proposals put forward that intend on solving this problem. Integrated System Health Management (ISHM) has thus far seen some growth in this sector, as a result of the extensive progress shown in demonstrating feasible and viable techniques. The problems related to these techniques were that they often consumed time and were too expensive and resourceful to develop. In this paper we present a radically novel approach for building prognostic models that compensates and improves on the current prognostic models inconsistencies and problems. Broadly speaking, the new approach proposes a state of the art technique that utilizes the physics of a system rather than the physics of a component to develop its prognostic model. A positive aspect of this approach is that the prognostic model can be generalized such that a new system could be developed on the basis and principles of the prognostic model of another system. This paper will mainly explore single switch dc-to-dc converters which will be used as an experiment to exemplify the potential success that can be discovered from the development of a novel prognostic model that can efficiently estimate the remaining useful life of one system based on the prognostics of its dual system.
\end{abstract}

Keywords-Prognostic Model; Integrated System Health Management (ISHM); Degradation; Duality; Cuk Converter

\section{INTRODUCTION}

Integrated System Health Management (ISHM) [1] is a future advancement incondition based asset management that attempts to create automated prognostic and diagnostic systems to maintain and improve the integrity and readiness expected from legacy Health and Usage Monitoring Systems. ISHM is functioned (over a certain period of time) to detect, predict, diagnose and mitigate adverse events caused by degradation, fatigue and faults in components. For instance, the following problems may occur during an important function related to a system's aircraft, regardless of whether the adverse event had been erupted by one of the subsystems. In order to diligently address this problem, it is important to develop technologies capable of integrating large heterogeneous distributed system [2] and asynchronous data streams from multiple subsystems; hence making it easier to detect a potential adverse event. The following technologies will later be used for diagnosing what caused the event, forecasting what consequences the event will have on the RUL of the system (i.e., whether the entire system will be put at risk), and lastly to take appropriate precautions to mitigate the event [1].

Moving on, in order to accurately estimate the remaining useful life of devices solely depends on developing prognostic models. This will require additional care and attention to be invested towards preparing the degradation profiles and establishing the physics of failure for every component. Therefore, it's necessary to gather and obtain the degradation profiles of every subsystem, including their individual components. This further result's as a new degradation profile being formulated for whenever a component is upgraded. This degradation profile is calculated from either analysing the accumulated damage or the data driven. A drawback of calculating the degradation profile is that any changes made in the design of the system willconsume time and incur additional costs, since the prognostics model will need to be re-upgraded. It's therefore safe to say that the proposals discussed above are very expensive and consume a lot of time to process while also being unreliable, noisy and inaccurate [3].

We intend to overcome these problems by developing a System - Level Reasoning (SLR) to at least provide the system with significant capabilities that can potentially reduce costs by adding a System Integrated Prognostic Reasoner (SIPR) to the system prognostics [1][4]. For Instance, a Vehicle Integrated Prognostic Reasoner (VIPR) is a project funded by NASA for developing the next generation VLRS. A typical functional module within the SLR is a System Reference Model. The 
System Reference Model divides the system into partitions. It also provides the necessary relationships between subsystems, which is required for the inference process. This partitioning allows the inference engine to reuse and link the same prognostic models to multiple subsystems and further minimize certification and qualification costs [1][4].

Although various methods and techniques including: neural network, fuzzy, statistics, semantic computing, graph theory, etc., have been thus far utilized for the development and enhancement of ISHM; however, ISHM continues to suffer from problems related to inefficient models, uncertainties and inadequate reasoning. Additionally, prognostic models also remain to be very costly and time consuming to prepare. The reason these problems exist is mainly because of the systems prognostics heavily relying on the physics of failure models and degradation profiles which are often considered to be inaccurate, inconsistent or even very noisy. We therefore believe that the ISHM system will greatly benefit if the prognostic of a component and system were to be perceived as a feature of a system rather than being perceived as the physics of components. The advantage of this approach is that it will enable SLR to develop prognostics for a new subsystem based on a collection of features (encompassing various models/patterns) already known from the previous prognostics of subsystems; hence saving a lot of time and resources. In order to successfully fulfil this task, SLR may need to employ various techniques associated with Soft Computing (SC),such as fuzzy and neural network within its Inference Engine and System Reference Model units, so that the subsystems properties can be linked to one another. In regards to this project, we expect that a duality connection will be found between the prognostics of dual systems, assuming that the prognostics of dual systems are seen as its parameters and features rather than physics of components.

The prognostics of the system shall be further explained in section 2. The principles of duality in electrical systems, along with brainstorming the duality concept of system's prognostics, are covered in section 3. Section 4 covers the prognostics of dc-to-dc converters with details of $\mathrm{Cuk}$ and its dual circuit. The proposed algorithm to develop prognostics for dc-to-dc converters using duality concept is presented in section 5 . Simulation results are discussed in section 6. Section 7 discusses future work. Lastly, section 8 concludes the major points discussed in the paper.

\section{PROGNOSTICS}

In condition-based maintenance, prognostics can be defined as a controlled engineering discipline that focuses on the prediction and estimation of the future course of a system or component that tries to establish when the system/component starts to slowly develop irregularities and faults to the point where it eventually malfunctions. A system or component that malfunctions means that it can no longer operate accordingly. The predicted lifecycle of a system or component is referred to as the Remaining Useful Life (RUL). RUL is used in decision making for contingency mitigation and maintenance. There are various scientific techniques used that help construct the prognostics of a system or component including: failure mode analyses, early detection of aging signs, and damage propagation models.Failure mechanisms are often used in conjunction with system lifecycle management to create prognostics and health management (PHM) disciplines. PHM is also sometimes known as system health management (SHM) or within the field of transportation applications; it is either known as vehicle health management (VHM) or engine health management (EHM). Building prognostic models constitutes of three main technical approaches which fall within the categories of data-driven approaches, model-based approaches, and hybrid approaches [1][4][5].

\section{A. Data-Driven Prognostics}

Data-driven prognostics [6] are mainly based on pattern recognition and machine learning approaches that help identify and detect trends and changes in the individual phases of a system's state. A way to predict trends in nonlinear systems is by using classical data-driven methods,such as stochastic models, an autoregressive model, the bilinear model, the projection pursuit, etc. Soft computing techniques that involve using various types of neural networks (NNs) and neural fuzzy (NF) systems have also been commonly adopted to deal with data-driven forecasting of a system state [7][8]. This prognostic approach applies to applications that havecomplicated system architecture, i.e., systems that incur high amount of cost when developing an accurate prognostic model. So by adopting this approach to deal with complex systems will lead the prognostics of a system to be much faster and cheaper to set up as compared to other approaches. Contrarily, data driven approaches may have a wider confidence intervals than other approaches which mean it will require a substantial amount of data for training purposes [9].

There are various strategies used to develop data-driven prognostics which involve the analysis of either (1) modelling cumulative damage and then extrapolating out to a damage threshold, or (2) directly learning from the data based on the remaining useful life.

As it is a lengthy and rather costly process to fail each and every system one by one, we thus seek to obtain the run-tofailure data which refers to the main fundamental setback, especially for new systems. In order to retrieve adequate datadriven prognostics, the accelerated aging data should be extracted cautiously from a number of similar/related products by using appropriate measuring tools. This means that both the quality and quantity aspects of the data driven prognostics will add to expenses; especially since the data sources may have been derived from a wide range of factors including: temperature, pressure, oil debris, currents, voltages, power, vibration and acoustic signal, spectrometric data, as well as calibration and calorimetric data. As a result, it is important to fully understand what parameters and signals will be necessary to measure, and which features will need be extracted from the noisy and high-dimensional data [6][7][9].

\section{B. Model-Based Prognostics}

Attempts made towards integrating a physical model of a system which is (either accomplished via micro or macro levels) into the estimated remaining useful life (RUL) is referred to as model-based prognostics [5]. The micro level (also known as material level) is often referred to as damage propagation model which is a physical model that is integrated 
in a series of dynamic equations. The following dynamic equations define the very relationships between damage and degradation of a system or component. They further define how the system or component operates under environmental and operational conditions. Despite it being almost impossible to measure many critical damage properties, an alternative solution would be to use sensed system parameters. However, it is possible that the level of uncertainty and inaccuracy to be increased. Even though uncertainty and inaccuracy is added as a result of sensed system parameters, uncertainty management would be considered with the realistic assumptions and simplifications, which may potentially overcome the limitations caused by the sensed system parameter [4][5][10].

In contrast to physical expressions used in micro-levels, macro-level models alternatively use mathematical models at a system level that help define the relationship among system input, system state, and system measurable variables. This mathematical model is often a simplified representation of the system. Simplification may lead to making prototyping faster; but the trade-off to this is that although the coverage of the model is increased, the accuracy of a particular degradation model is consequently decreased. In addition, within a complex application, such as a gas turbine engine, there would be a lack of knowledge in attempting to develop the proper mathematics for all subsystems or components. Again, this leads to uncertainty and inaccuracy, similar to micro-level models; which means simplifications must be considered by performing uncertainty management procedures [1][4][10].

\section{Hybrid Approaches}

In reality, it is almost impossible to either have a purely data-driven or purely model-based approach. However, both these models do share parts of one another's mechanisms. The intention of hybrid approaches is to show the strength of both 'data-driven' approaches and 'model-based' approaches into one prognostic strategy. Two well-known categories of Hybrid approaches are, 1) Pre-estimate fusion and 2) Post-estimate fusion. The first technique applied, hardly has any 'ground truth' data or 'run-to-failure' data available. The second technique is fitted for situations where uncertainty management is required. This means that the second technique helps to narrow down uncertainty intervals of data-driven or modelbased approaches while also improving accuracy [11][12].

\section{PROGNOSTICS OF DUAL SYSTEMS}

Duality is one of the fundamental properties which can be consistently seen in physical systems, such as, electrical, mechanical systems, etc. [13][14]. It has an interesting history in mathematics, engineering and science. Duality relations have been identified between geometric objects, algebraic structures, topological constructs and various other scientific constructs. In regards to electrical systems, duality relations have appeared in the core principles for any theorem within an electrical circuit analysis, for situations where there is a dual theorem that replaces one of the quantities with dual quantities.

Examples of such dual quantities in electrical systems are current and voltage, impedance and admittance, meshes and nodes found in electrical systems (shown in Table 1) [15].
TABLE I. DUALITY PRINCIPLE IN ELECTRICAL SYSTEMS

\begin{tabular}{l|l}
\hline \multicolumn{1}{c|}{ System } & \multicolumn{1}{c}{ Dual of System } \\
\hline Voltage of nodes or across device & Current of branch or mesh \\
\hline Current of branch or mesh & Voltage of nodes or across device \\
\hline Resistor (R) & Conductivity (1/R) \\
\hline Capacitor (C) & Inductance (C) \\
\hline Inductance (L) & Capacitor (L) \\
\hline Voltage Source (Vs) & Current Source (Vs) \\
\hline Current Source (Is) & Voltage Source (Is) \\
\hline Kirchhoff's Current Law & Kirchhoff's Voltage Law \\
\hline Kirchhoff's Voltage Law & Kirchhoff's Current Law \\
\hline Mesh/Loop & Node \\
\hline
\end{tabular}

In regards to duality concepts, a duality relationship between two electrical circuits is expected to be found, if the values of the parameters and topologies of both circuits are linked to one another based on details in Table 1. Looking at it from a mathematical perspective, dual circuits are known to have the same mathematical model, apart from their parameter differing. Even though the function of systems are different, their prognostics still can be assigned to each other on the basis of dual relationships found between the systems, along with having the same mathematical model with dual parameters shown in Table 1. This provides us with the required facilities to develop the prognostics of a system based on the prognostics of its dual system.

Graph theory [13] well established that the behaviour and the functionality of a system can be recognized by knowing the topology of a system without having to know the components and devicesused in the system (considering we already know the nodes voltages and currents of the branches in the circuits). It can thus be expected that graph theory provides us with the capability to construct the prognostic of a system based on its topology rather than concentrating on the integrated devices and components within the system. It is also to be expected, systems that share similar or dual topologies and mathematical models will also share similar prognostics regardless of the components integrated within the system. This makes it possible to investigate how prognostic models can be constructed from knowing the topology of system rather than having to know thephysics of failure of a system. This therefore makes the process of modelling the prognostics of a system much more feasible and realistic, as it saves a substantial amount of resources and time, since you wouldn't have to go through the process of individually testing every system in order to identify its prognostics.

Fig. 1 shows an example of dual circuits. Using Kirchhoff's laws, it can be said that both circuits have the same mathematical model as shown in equation 1 for circuit in Fig. 1-a; and equation 2 for circuit in Fig. 1-b:

$$
\begin{gathered}
\text { Va. }\left(1 / R_{1}+1 / R_{2}+1 / R_{3}\right)=0 \\
\text { Ia. }\left(R_{1}+R_{2}+R_{3}\right)=0
\end{gathered}
$$

If for instance a degradation mechanisms is added, $R_{2}$ in circuit of Fig. 1-b is aged towards a short circuit $\left(R_{2} \rightarrow 0\right)$, this is turned as $\left(1 / R_{2} \rightarrow \infty\right)$ in circuit of Fig. 1-a. 
In reality, this represents the duality principles shown in Table 1 which proves that the resistor is a dual of a conductive; or in regards to this example, it can be known since the short circuit is a dual of an open circuit.

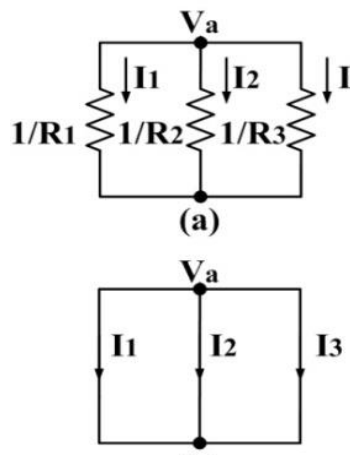

(c)

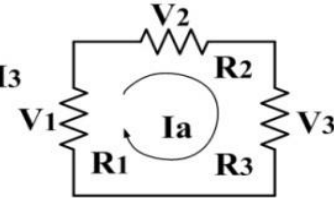

(b)

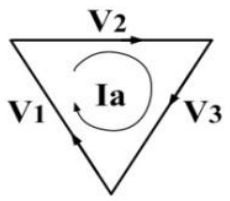

(d)
Fig. 1. a, b) Resistive circuit with duality relationship, c,d) Graphs for circuits $1-\mathrm{a}$ and $1-\mathrm{b}$.

The same rules can be applied to more complicated circuits where various components, including capacitors and inductances are also used. The most critical point that needs to be taken into account is the fact that degradation and failure mechanisms of dual components are not truly related to one another. For instance, the degradation mechanism of capacitor is not in any shape or form related to degradation mechanisms of inductance.

A way to deal with this problem is to rely on the wellknown physics principles, such as Ohm's and Kirchhoff's laws. In reference to these two laws, it can confidently be said that any electric component can be formulated by using voltage across the component and current through the component. Alternatively, in regards to graph theory's basic principles of circuit and system design, it has been well known that the behaviour of a system is fully formulated if the voltage of all nodes and current through all the branches in the circuit are also known. This means that behaviour and the function of circuit can be fully formulated no matter what components are used in the circuit, as long as all the voltages and currents are known Fig. 1-c and 1-d, respectively show the graph of the equivalent circuits in Fig. 1-a and 1-b.

Perceiving it from a circuit level, the details required for the development of a prognostics model for a circuit does not necessarily need to be known. Essentially, sensors are used to measure voltages, currents, temperature, etc. By basing it on the meaning of the sensed values, allows the experiences of a degraded circuit or system of any form, to be interpreted as a circuit not functioning properly. Although this principle can be applied for greater purposes, i.e., to design a device independent prognostic model, this paper will for now mainly concentrate on presenting a realization of duality principles for the development of prognostics for dual circuits.

In addition, duality concept has already been recommended for diagnosing faults. Reference [16] proposes a fault diagnoser based on the duality principle and the optimal control theory for linear systems. However, this paper will present duality applications in system prognostics.

\section{PROGNOSTICS OF DC-TO-DC CONVERTER}

A basic building block for power convertor type systems is dc-to-dc converter. There are many dc-to-dc voltage and current converters that have various topologies. These topologies can be defined algebraically [17]-[20], graphically, [21][22] or in a matrix form [23]-[25]. It is of significant interest in unifying converter topological characteristics, relationships, and analysis [26]. In regards to health management, the aim would be to develop a basic structure, model or concept that shows where all the converters, including their prognostics may have been derived and mapped. This unified model can lead to many advantages in developing conditional based monitoring, and System- Level Reasoning (SLR).

The underlying concepts related to basic converters can be unified on the basis of what has been already presented in [27] with regard to duality principles and in relation with current and voltage-source converters. The authors in [27] used circuit transformations to unify the basic converters, ultimately showing that other converters are derivable transformation topologies of the basic converter.

This section shows how duality concept can be used to develop prognostic models for Cuk converter and its dual circuit. The following simulations were all conducted with ORCAD and MATLAB. Schematic of Cuk converter and its dual circuit are shown in Fig. 2-a and 2-b.
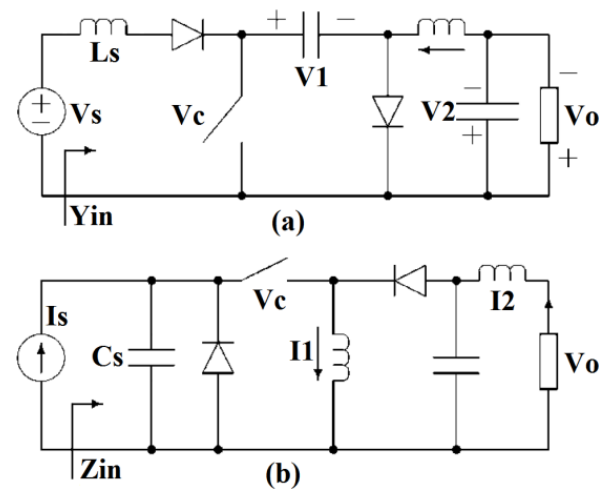

Fig. 2. a) Cuk Converter, b) Dual circuit for Cuk converter in 2-a.

We use certain values for Cuk converter devices, as well as all the equations depicted in reference [28] for all the simulations in this paper. Cuk is a step-down/step-up converter that shares a similar switching topology with buck-boost converter. It thereby presents the voltage ratio of a buck-boost converter [28]:

$$
V_{o} / V_{s}=D_{s} /\left(1-D_{s}\right)
$$

where $v_{o}$ is output voltage, $v_{g}$ is the input voltage, $D_{s}$ is the duty cycle of the switch $\mathrm{t}_{\mathrm{on}} /\left(\mathrm{t}_{\mathrm{on}}+\mathrm{t}_{\mathrm{off}}\right)$; and ton and toff are durations for when the switch is on and off. Equation 3 is calculated from the principle of conservative energy and the fact that the inductor currents relate to the input and output currents. This equation shows that the output voltage can be controlled by maintaining the duty cycle of the switch. 
Basedon the type of switching scheme, output voltage can be either higher or lower than the input voltage. The state equations for Cuk converter are:

$$
\begin{gathered}
x^{\prime}=A x+B v_{g}+B_{c} d \\
v_{o}=C_{x} \\
x=\left[\begin{array}{llll}
v_{2} & v_{1} & i_{2} & i_{1}
\end{array}\right]^{\prime}
\end{gathered}
$$

The Cuk converter has two inputs, a control input $\left(\mathrm{V}_{\mathrm{c}}\right)$ and an input from the power supply $\left(\mathrm{v}_{\mathrm{s}}\right)$ and one output $\left(\mathrm{v}_{\mathrm{o}}\right)$. Therefore, matrix [A B C D] relates to 'state space matrices' for the open-loop model from the $\mathrm{v}_{\mathrm{s}}$ to the $\mathrm{v}_{\mathrm{o}}$. Similarly, [A $\mathrm{B}_{\mathrm{c}}$ $\mathrm{CD}$ ] is the state space matrices from the control input $\mathrm{d}$ to the output $\mathrm{v}_{\mathrm{o}}$. Values for $\mathrm{A}, \mathrm{B}, \mathrm{B}_{\mathrm{c}}, \mathrm{C}$, and $\mathrm{D}$ are given in [17]. The same equation can be extracted for dual circuit of Cuk converter in Fig. 2-b; however, parameters are used in a dual form as shown in table 1. Switches in Fig. 2 are IGBT with a control voltage $\mathrm{V}_{\mathrm{c}}$. $\mathrm{Y}_{\text {in }}$ and $\mathrm{Z}_{\text {in }}$ are input admittance and input impedance of Cuk circuit and its dual circuit.

Inside converters, the components that are mainly damaged refer to the IGBTs and capacitors. Alghassi et al has discussed different failures mechanisms related to IGBT and they have also presented prognosis model for the dominant failure at a component level in [29][30]. IGBT experiences a number of failure mechanisms including: bond wire fatigue, bond wire lift up, corrosion of the wires, static and dynamic latch up, loose gate control voltage, etc. The resulting affects mentioned are too complex, but we assume that these failure mechanisms can cause IGBT to behave as either an open circuit on a collectoremitter or a device encountering malfunction on its gateemitter control. For instance, IGBTs thermal junction is increased due to solder crack which turns to wire bond lift off that increases the resistor relating to the collector-emitter. On the other hand, hot carrier injection is increased due to electrical stress. This causes short circuit on the IGBTs gateemitter junction. The result of this failure, leads to IGBT's gate controllability being missed (loose gate control voltage) causing IGBT to malfunction. The result of this effect is an increase in current through collector-emitter which means that the resistor of collector emitter is decreased. Therefore, it can be established that wire bond lift off and loose gate control voltage are failure mechanisms that presents some kind of duality relationship. While one of them increases the resistor, the other one decreases the resistor. Generally, we assume that IGBT's failure and malfunction mechanisms are parameters that have duality relationships.

Fig. 3 shows IGBT run to failure data relating to four different IGBTs. This data is very noisy and needs to be filtered, but there are still a number of states that can be seen in the data. These states refer to cracks or wires that were lifted up due to degradation mechanisms. The resulting effects are changes in the IGBT's functionality; and changes in the channel resistor of that IGBT. We assume that degradation is processed in a form of duality for Cuk and its dual circuit, so that if IGBT of Cuk experiences degradation towards its open circuit, IGBT of dual circuit of Cuk is degraded towards short circuit.
By the time that the IGBTs are damaged, $\mathrm{C}_{\mathrm{s}}$ and $\mathrm{L}_{\mathrm{s}}$ are fully charged, as well as the other energy storage components lose energy, so $\mathrm{V}_{\mathrm{o}}$ would be 0 . It is, however, impossible to have a real short circuit in IGBT, thus we assume that it may have happened when the current through the collector-emitter exceeds over its limit just before the IGBT is burned out.

Based on the level of accuracy, there are number of models that can be applied to a real capacitor and an inductance. To simplify a simulation, we assume that the capacitor and the inductance can both be modelled like Fig. 4 for the purposes of this paper. These models will present duality relationship between capacitance and inductance while also presenting the energy lost by the resistors. $\mathrm{R}_{1}$ typically has had very large values, while $R_{2}$ has a small value; but due to degradation, these resistors are changed towards either open or short circuits.

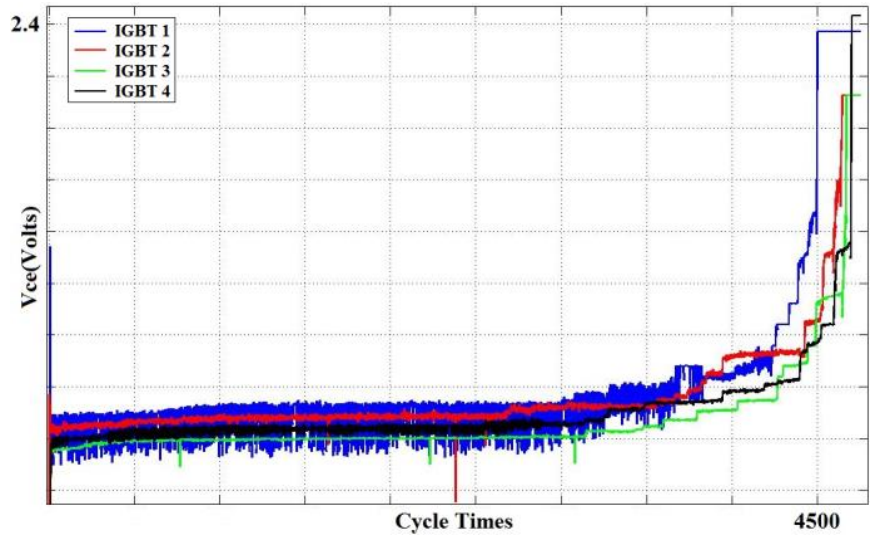

Fig. 3. Real model for a) Capacitor, b) Inductance.
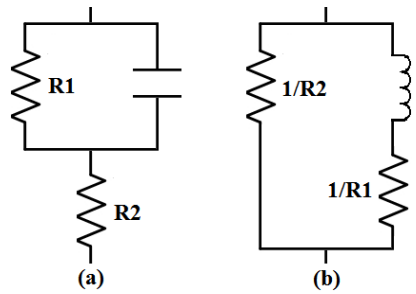

Fig. 4. Real model for a) Capacitor, b) Inductance.

\section{AlgORITHM TO DEVElop PROGNOSTICS}

Fig. 5 illustrates the proposed algorithm devised to develop this prognostics model. The same process that possesses different sets of run to failure degradation and malfunction profiles is repeated for both Cuk and its dual circuit. At first the components of the circuits are set to be in a good condition. Then as soon as the time step for the circuit is increased, the values of the components are changed by using a series of values provided within the degradation profile for the new time step. Signals, such as $\mathrm{v}_{1}, \mathrm{v}_{2}, \mathrm{v}_{\mathrm{o}}, \mathrm{i}_{1}, \mathrm{i}_{2}, \mathrm{i}_{\mathrm{o}}$, are measured at each time step phase.

The following signals are used for calculating the properties of the system, such as transfer functions, input and output impedances and admittances. Subsequently, the system degradation is turned according to changes it has encountered during the transfer functions $\left(Z_{c}(d, t), Y_{c}(d, t), Z_{d c}(d, t), Y_{d c}(d, t)\right)$. 
So where $\mathrm{d}$ is an index of a selected degradation profile, $\mathrm{c}$ is Cuk and dc is the dual circuit of the Cuk converter. Whenever $\mathrm{d}$ is altered, time step (t) is reset to zero which resets the process of the circuit to a healthy condition for the new degradation scheme.

The reason for measuring the mentioned signals and parameters is that it would make it possible to understand how energy is transferred between capacitances and inductances; and how that transferred energy is lost when the system is also degraded.

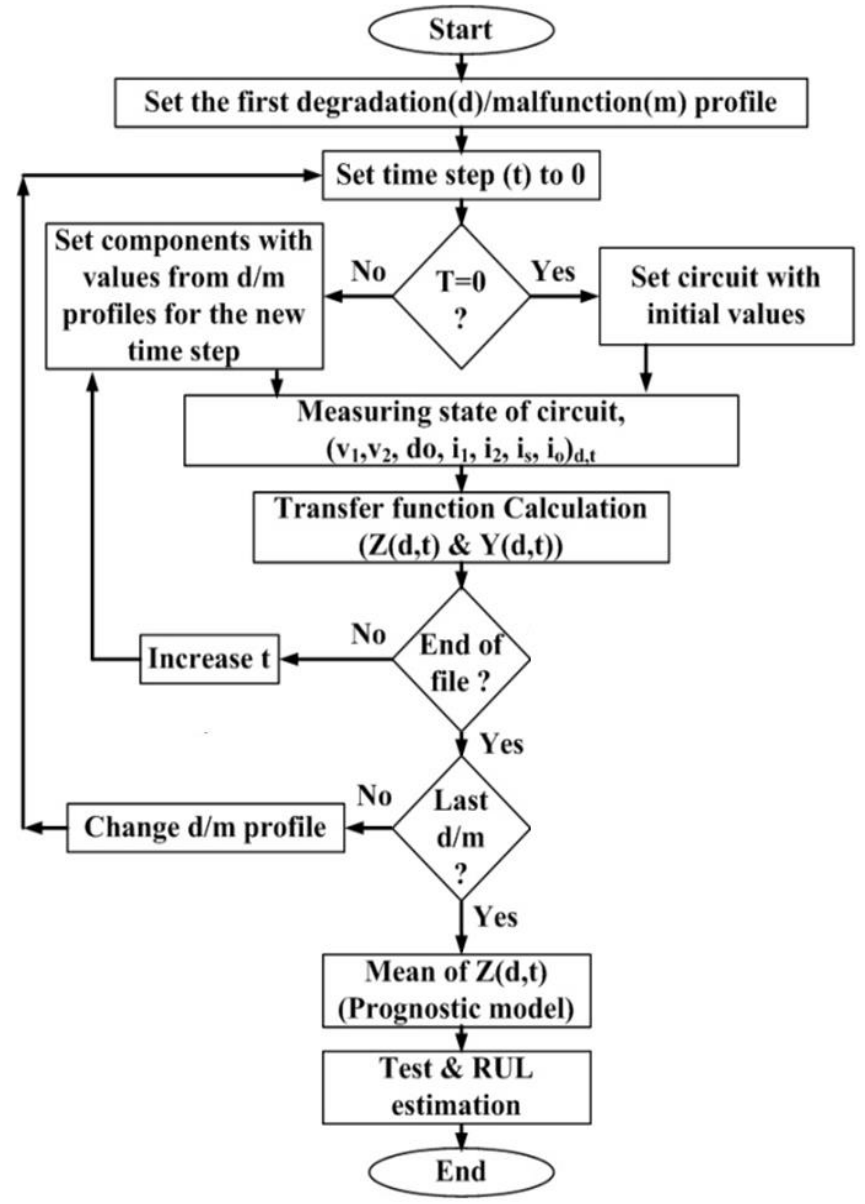

Fig. 5. Algorithm used to develop prognostic model. $\mathrm{d} / \mathrm{m}$ : degradation/mulfunction number; t: time step or cycle.

To successfully estimate the remaining useful life of both converters, the same process is to be repeated for numbers of different degradation and malfunction profiles. The implication of such a process is to obtain standard Tee and Pi models for Cuk converter and its dual circuit, as shown in Fig. 6. This means that there would be a number of time dependent Tee and Pi models, one for each degradation and malfunction profile. There are many different techniques, such as neural network, fuzzy, statistics, etc., that can be utilised in order to generate a universal prognostic model for the converters (Cuk and its dual) out of all time dependent Tee/Pi models needed to be trained. Here, we just use a mean value to simplify and speed up the process.
The resulted time dependent transfer function which is known as prognostic model is excited with step function $(\alpha \mathrm{u}(\mathrm{t}))$ during the RUL estimation. Step function $\alpha \mathrm{u}(\mathrm{t})$ provides a fixed input of $\alpha$ for the converter over the period of $t>0$. We later assign fuzzy values to the output of transfer function excited with $\mathrm{u}(\mathrm{t})$. The fuzzy values represents whether there are a small, medium, normal, transient and big changes experienced at the output of the converter.

The term 'normal' in the fuzzy set, means that changes in the signals can be ignored and transient means that the circuit is in a transient mode and should be settled in a steady state during a specific time constant. RUL is estimated using the MAX fuzzy function which is applied on the triggered fuzzy values. MAX fuzzy function selects the maximum fuzzy value among the fired membership functions.

During this process, RUL is estimated in a fuzzy form, and therefore needs to be de-fuzzified. During the de-fuzzification step, RUL is also scaled up, so that the integration of the estimated RUL (in fuzzy form) reflects the maximum life cycle of the circuit, Fig. 7. Confidence levels are implemented using fuzzy adjectives and adverbs found in fuzzy base knowledge and fuzzy rules.

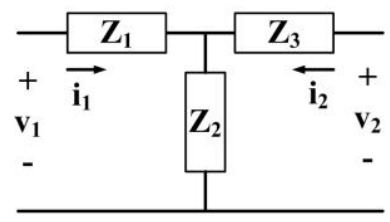

(a)

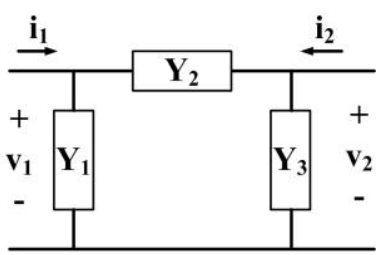

(b)
Fig. 6. Tee and Pi Models

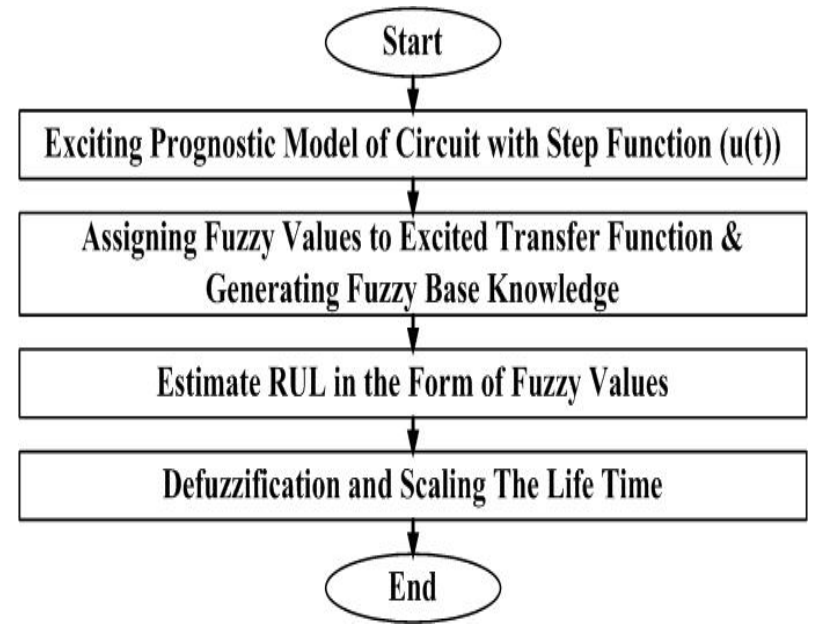

Fig. 7. Simplified Algorithm for RUL Estimation.

\section{Silumation RESUlts AND Discussion}

Simulation results, using ORCAD 16.6 that reflect the circuits shown in Fig. 3 are presented in Fig. 8 and 9. Looking at these figures, it is apparent that $\mathrm{V}_{\mathrm{ol}}$ has the same trend as Io2; and the same for Iin1 and $\mathrm{V}_{\text {in2 }}$. 
These in turn reflect the similarities encountered within the transfer functions, such as $\mathrm{Z}, \mathrm{Y}, \mathrm{A}_{\mathrm{v}}$ and $\mathrm{A}_{\mathrm{i}}$ shown in Fig. 10. As shown in Fig. 11, we used the IGBT model for our simulations. To add degradation to our simulations, we changed the IGBT's parameters, such as $R_{\text {on }}$ in such a way that a trend of failure in Fig. 2 will be obtained from the IGBT model in Fig. 11.

To speed up the simulation, we intend to have all 4500 cycles shown in Fig. 2 in just $25 \mathrm{~ms}$. The result from this mapping is that the degradation will be accelerated in such a way that the first degradation will be experienced around $8 \mathrm{~ms}$ after exciting the circuit with step function, $\mathrm{u}(\mathrm{t})$; however, the threshold needed to estimate whether the IGBT has aged enough to incur damage in an earlier time is around $10 \mathrm{~ms}$. The same life time and threshold can be expected from energy transfer and power of $\mathrm{C}_{\mathrm{o}}$ and $\mathrm{L}_{\mathrm{o}}$, respectively shown in Fig. 12 and 13 for Cuk and its dual circuit.

The figures illustrate that as a result of degradation, energy is not sufficiently transferred in the circuit. So this informs us that the health state of a circuit can just as well be understood from the state equations of a circuit, whereby energy signals from storing elements, capacitors and inductors are used as state variables.
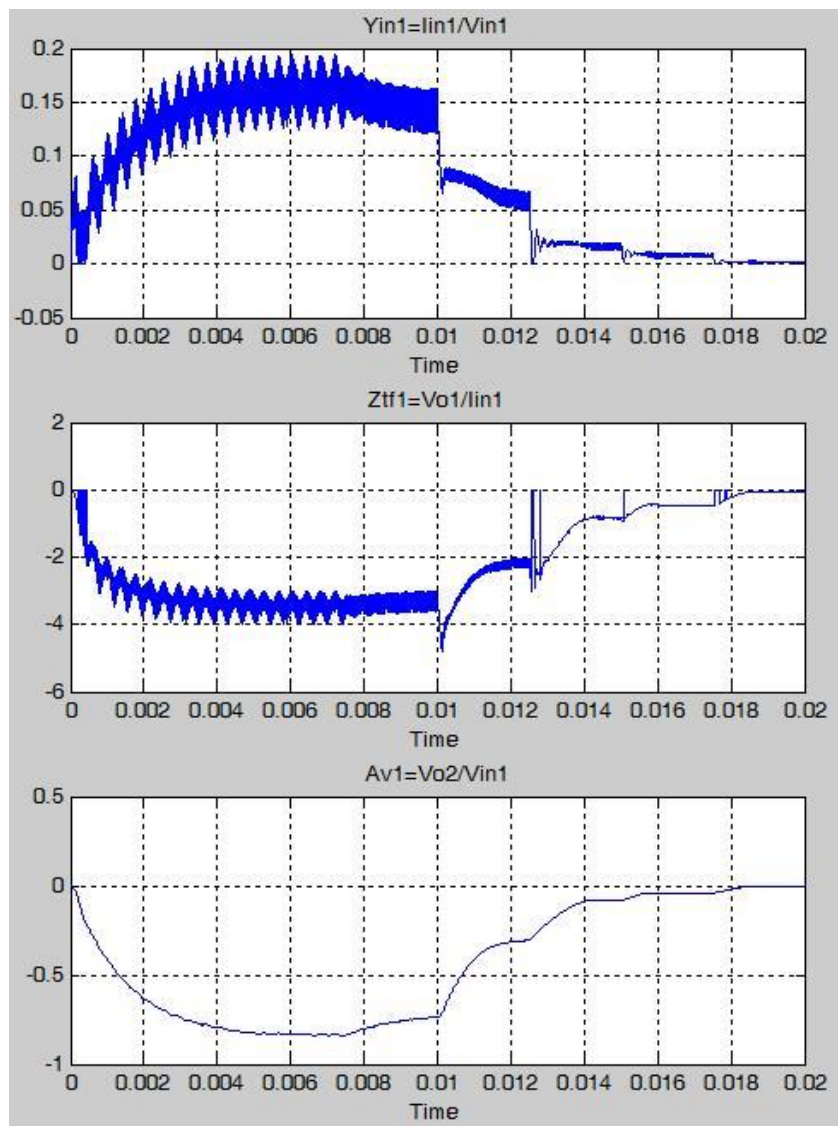

Fig. 10. Changes in transfer functions due to degradation.

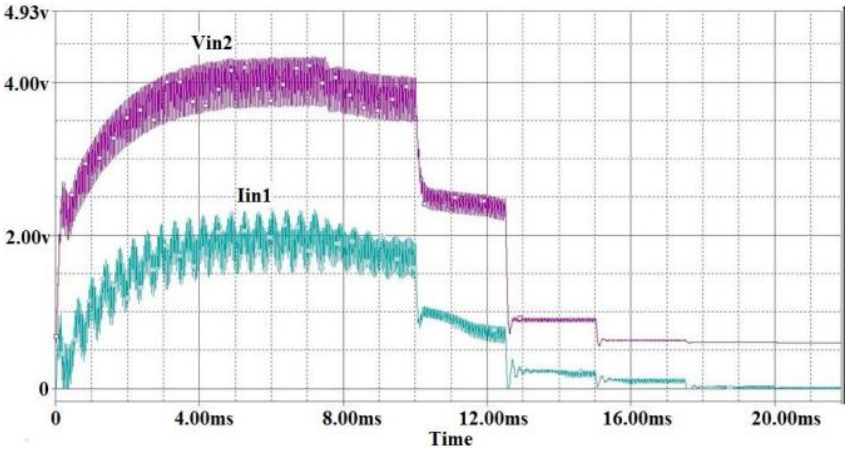

Fig. 8. Changes in Iin1 and Vin2 due to degradation in IGBTs.

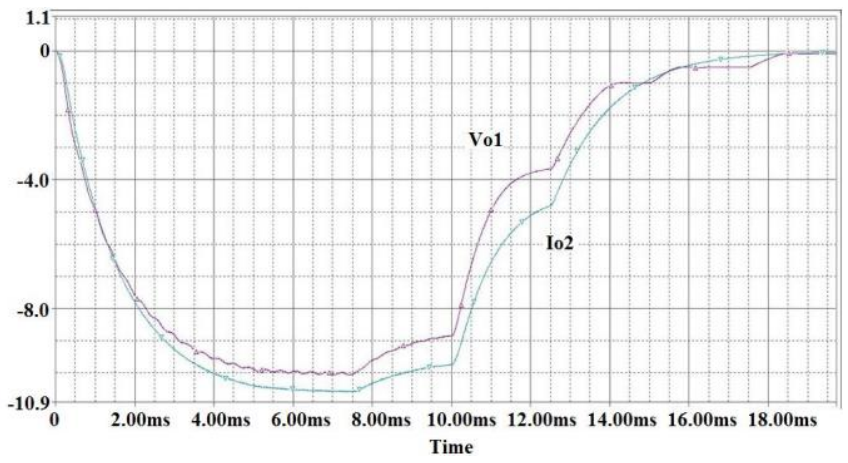

Fig. 9. Changes in Vo1 and Io2 due to degradation in IGBTs.
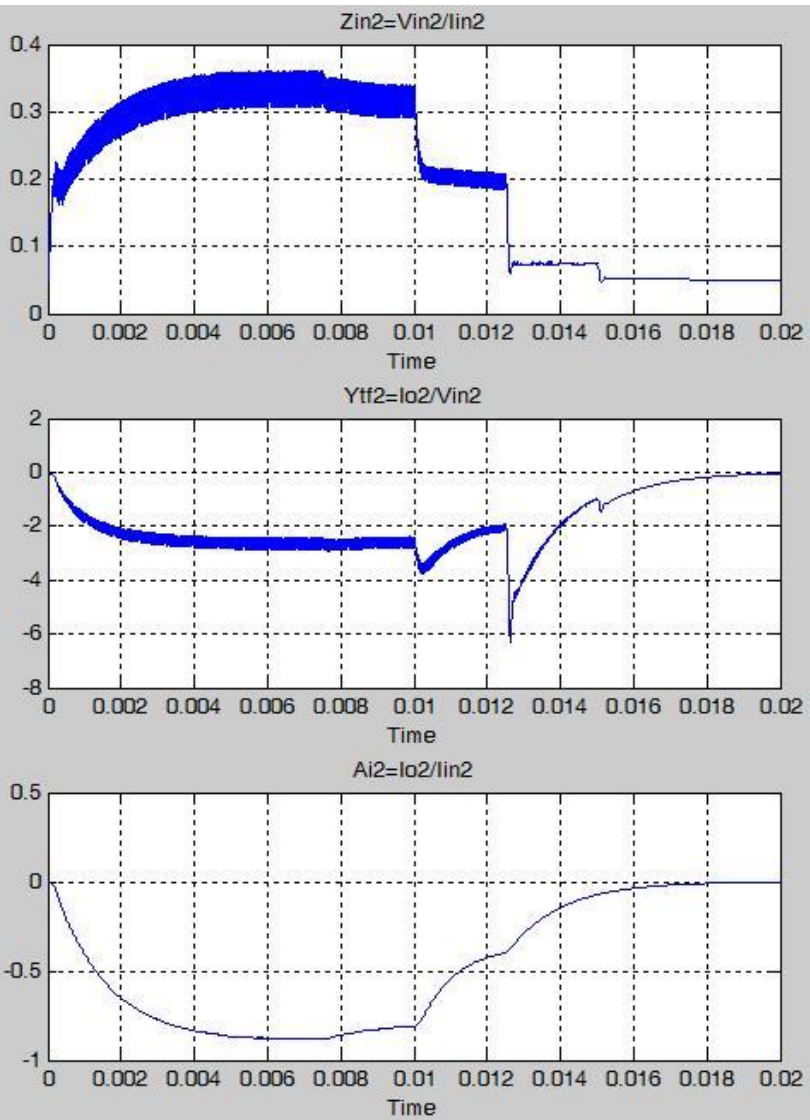


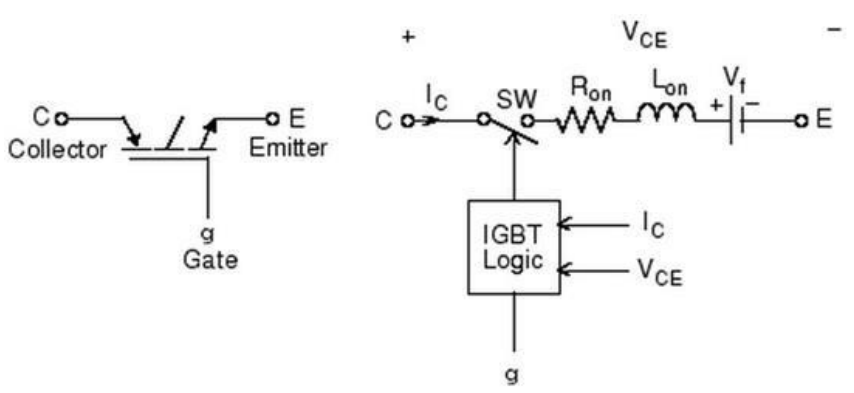

Fig. 11. IGBT Model for Simulation.

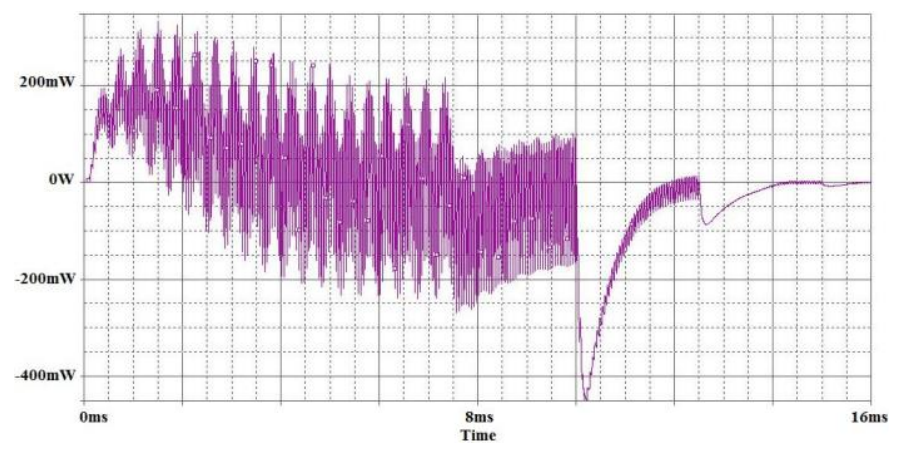

Fig. 12. Energy in $\mathrm{C}_{\mathrm{o}}$ of Cuk converter in Fig. 3.

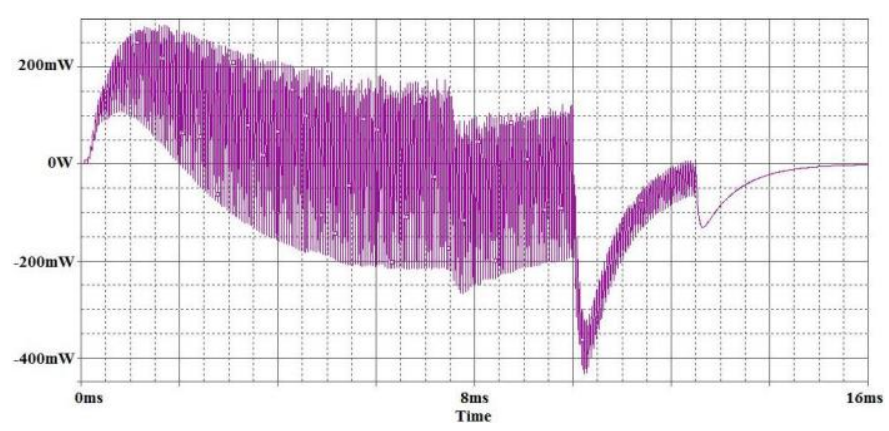

Fig. 13. Energy in $L_{o}$ for dual circuit of Cuk in Fig. 3.

The timings presented here are not realistic as we accelerated the aging mechanisms in the simulation. Nevertheless, it does clearly prove that the concept of the circuits with duality relationships or even with similarities in components and topologies may also have similar prognostics models.

Every signal, energy and transfer function monitored in the figures have similar information with regard to the effects of degradation of a circuit. However, some of these parameters are rapidly changing due to the switching scheme of IGBT and energy transfer in Capacitors and Inductors, but in most cases the same trend can be found in all these signals. This experiment only refers to the degradation profile concerning the IGBT which refers to the component that mostly experiences degradation during real time; while simultaneously all other components are assumed to behave as non-aged devices (in all simulation). As shown in Fig. 10, $\mathrm{A}_{\mathrm{v} 1}$ and $\mathrm{A}_{\mathrm{i} 2}$ seem to be the best for RUL calculation. Other transfer functions and signals are viewed as noisy data, thus requiring further care to be conducted, such as filtering in order to reduce uncertainties for accurate RUL estimation. For instance, instead of making direct decisions based on monitored signals, the monitored signals can be shifted in the FIFO (First-In FirstOut stack) one by one, and the mean value of available data in the FIFO could be hence used for the RUL estimation. FIFO has a fixed storage length, so that shifting a new sample to the FIFO will release the sample that had been already shifted into the stack at the earliest time. Mean value of FIFO captures the trend of signals and eliminates noise, unwanted information and uncertainties. The following FIFO will increase system reasoning within RUL estimation. The length of FIFO has had a great impact on eliminating noise, but it normally shouldn't take that long to lose trend of system degradation. Additionally, implementing a mean value on the stored data in the FIFO may add DC value (i.e., mean value) to the estimated RUL. As DC value is constant (i.e., meaningless information), it will make it easier to eliminate the DC value from RUL.

In order to simplify the process, we use $\mathrm{A}_{\mathrm{v} 1}$ and $\mathrm{A}_{\mathrm{i} 2}$ for the RUL estimation using fuzzy logic techniques to estimate the remaining life time of circuit, as shown from the algorithm in Fig. 7. All the input and output membership functions are set in Gaussian form with input fuzzy values as \{small, medium, normal, transient and big $\}$ and output fuzzy values as $\{$ health_state_1, health_state_2, health_state_3, health_state_4 and health_state_5\}. Fuzzy values at input refer to the changes in the trend of $A_{v 1}$ and $A_{i 2}$. Fuzzy values at output refer to the life state of circuit, such as young for health_state_1 and, aged for health_state_5. We also use number of adverbs and adjective to address $10 \%$ and $90 \%$ confidence levels in life estimation. Fig. 14 shows the final RUL in fuzzy form. This figure shows how the circuit behaves in different life/health states during its life cycle. $\mathrm{LF}_{1}$ to $\mathrm{LF}_{5}$ show the life domain of each health state. $\mathrm{LF}_{1}$ comes from having huge transient period at the beginning of $\mathrm{A}_{\mathrm{v} 1}$ and $\mathrm{A}_{\mathrm{i} 2}$. In reality, we are not faced with such a big transient period, but it is included in our simulation just because of the acceleration in the degradation process. $\mathrm{LF}_{2}$ represents the how long the system works without the inclusion of degradation, and the rest refer to durations to which the circuit does experience degradation. The reason LF4 appears twice is that the circuit is not experiencing big changes in its transfer functions, but there are meaningful transient periods found that split the LF4 into two separated Gaussian fired fuzzy values.

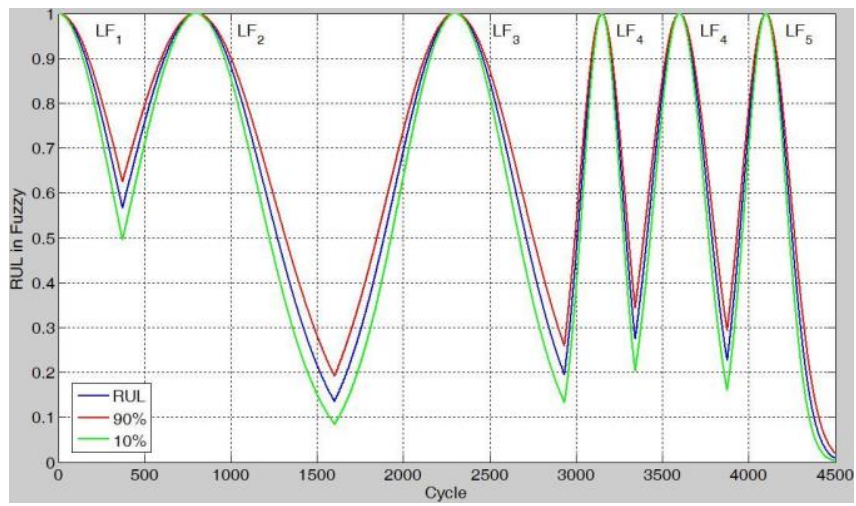

Fig. 14. Resulting RUL after testing prognostic model with data test. 
The results reflecting the prognostic model is tested with an additional degradation profile. This will be handled as a test data which will assist us in estimating the remaining useful life time for the converter. Fig. 15 shows the de-fuzzified RUL that represents the remaining useful life with $10 \%$ and $90 \%$ confidence levels. Ideally, it is expected that the life of a circuit is decreased as a negative ramp in Fig. 15; however, our simulation shows that the RUL is slightly wavy.

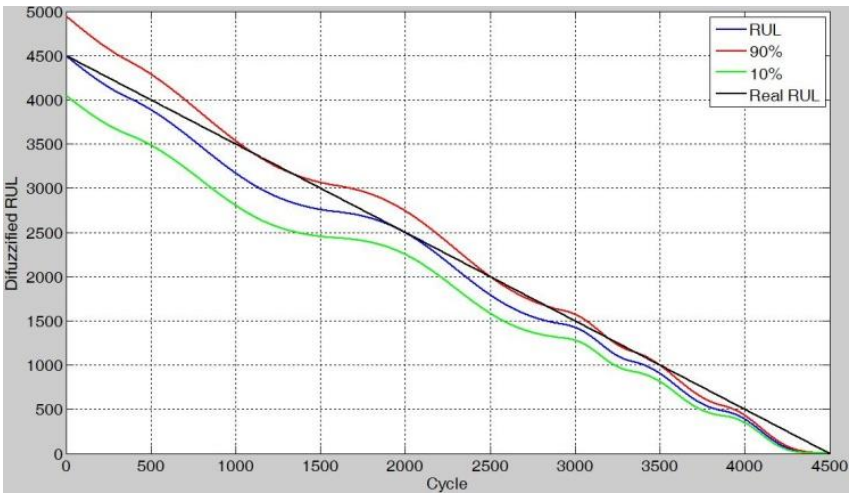

Fig. 15. Resulting RUL after testing prognostic model with data test.

We realized that if a degradation profile is used for Cuk, such that it's converted to a malfunction profile for its dual circuit so that the IGBTs in both circuits always remain in dual forms; then a duality relationship would be found between the transferred functions of these two circuits. For instance, $Z_{c}(t)$ is equal to $\mathrm{Y}_{\mathrm{dc}}(\mathrm{t})$. This is because as the degradation profile changes the IGBT of Cuk towards an open circuit; its malfunction profile also changes the IGBT of dual circuit towards a short circuit.

If the malfunction profile for dual circuit of Cuk is not extracted from the degradation profile of a Cuk circuit, then $\mathrm{Z}_{\mathrm{c}}(\mathrm{t})$ is not identical to $\mathrm{Y}_{\mathrm{dc}}(\mathrm{t})$. However, we come to a conclusion that if the whole process is repeated for number of different degradation and malfunction profiles and that the mean value of $\mathrm{Z}_{\mathrm{c}}(\mathrm{t})$ and $\mathrm{Y}_{\mathrm{dc}}(\mathrm{t})$ are used for comparison; leads to meaningful similarity patterns to be found between $Z_{c}(t)$ and $Y_{d c}(t) . Z_{m c}(t)$ can be used for the mean value of $Z_{c}(d, t)$ and $Y_{m d c}(t)$ can be used for the mean value of $Y_{d c}(d, t)$, in situations where $m$ refers to the mean value. $Z_{m c}(t)$ and $Y_{m d c}(t)$ can be both used as prognostic models for Cuk and its dual circuit. However, these two transfer function are not exactly identical, but they would be more similar to one another if the process that is required to be executed to obtain the functions is repeated for various numbers of degradation and malfunction profiles for both circuits. By implementing more intelligent algorithms that use stochastic, neural network, fuzzy and other techniques instead of a simple mean value function will increase the accuracy of this prognostic model. Implementing such intelligent algorithms also reflects the future aim and direction of our research. Additionally, we should be aware that prognostics have always been a way to estimate the life time of devices and systems within different confidence levels. Confidence levels provide assurance, so that we can comfortably rely on the performance of an aged system. The point is the accuracy of prognostic models has always been under doubt and remains to be under margins of confidence levels. In summary, by using the prognostic model of a system for other systems where similarities in their properties (like duality) are found, would give us a more accurate and reliable representation of the state and condition of the system. This is assuming that the prognostics are developed from adequate number of degradation profiles, and that they also have the right minimum and maximum confidence levels.

\section{FUTURE WORK}

In this paper, we have looked at the IGBT in a converter as a critical component, thus meaning that the life expectancy of the converter is dependent on the remaining useful life of the IGBT. However, as for another component, such as a capacitor, it is also susceptible to thermal and mechanical stress. Thereby we must investigate whether it is classed as a dominant component failure in a converter or not. So in order to improve the novelty of duality in prognosis, requires one to have a cluster of components. This may overall have a remarkable impact on developing ISHM for critical applications.

\section{CONCLUSION}

In conclusion, this paper shows that the prognostics of systems that share similar properties in the form of duality can be applied to one another. A prognostic model is developed in the form of a time dependant transfer function where based on the degradation mechanisms related to a system's components, the values are subsequently altered over a certain period of time. So by having the prognostics assigned to a system's property will thereby reflect the duality connection found within the degradation and malfunction profiles of a system. So if we were to consider that the components of a system are aged, this will mean that their dual components in the dual circuit will befaced with malfunction.

The accuracy of the developed prognostic model is highly dependent on the number of degradation profilesavailable; and the methodology used to train the time dependant transfer function. The minimum and maximum confidence levels are used to guarantee and express the accuracy of this model. However, this approach is presented just for Cuk converter and its dual circuit, but it seems that the same technique can be used for systems that have slightly similar mechanisms, properties topologies and degradation. Thereby, further research needs to be conducted for systems that are not in dual forms, especially for the purposes of exploring how the prognostic model of a system could be mapped to the prognostic model of another system.

The advantage and usage of such a technique is emphasized in the implementation stage of the inference engine for SystemLevel Reasoning (SLR) and System Integrated Prognostic Reasoner (SIPR). It additionally provides us with the required facility to transfer degradation knowledge and experiences between systems. This means that the development of prognostics for huge systems, such as heterogeneous distributed systems used in applications like aircraft will be much faster, while decreasing the cost assigned to accelerated aging tests and preparing degradation profiles. We ultimately intend on pushing forward with our research, in order to apply this technique to the development of the prognostic inference engine and reasoned for aircraft. 


\section{ACKNOWLEDGMENT}

The authors would like to sincerely thank Professor C Mark Johnson and Dr Paul Evans from the Power Electronics, Machines and Control Group, University of Nottingham for the contribution of failure data of the IGBTs and the power cycling test rig configuration.

\section{REFERENCES}

[1] I. K. Jennions, "Integrated Vehicle Health Management Perspectives on an emerging field", SAE International, Warrendale, pennsylvania, USA 2011, pp. 100-110.

[2] A. El-Sayed and M. El-Helw, "Distributed Component-Based Framework for Unmanned", Proceeding of the IEEE International Conference on Information and Automation Shenyang, China, June 2012, pp. 45-50.

[3] W. Wenbin and M. Carr, "A Stochastic Filtering Based Data Driven Approach for Residual Life prediction and Condition Based Maintenance Decision Making Support" Prognostics \& Systems Health Management, IEEE Conference, Macao, China, Jan. 2010, pp. 1-10.

[4] I. K. Jennions, "Integrated Vehichle Health Management The Technology”, SAE International, Warrendale, pennsylvania, USA, 2013, pp. 139-154.

[5] M. Daigle and K. Goebel, "Model-Based Prognostics under Limited Sensing", IEEE Aerospace Conference, Big Sky Resort, USA, March 2012, pp. 1-12.

[6] C. Chen and M. Pecht, "Prognostics of Lithium-Ion Batteries Using Model Based and Data-Driven Methods", 2012 Prognostics \& System Health Management Conference, IEEE PHM Conference, Beijing, China, May 2012, pp. 1-6.

[7] H. Chao, D. Byeng, K. Youn, and K. Taejin, "Semi-Supervised Learning with Co-Training for Data-Driven Prognostics", Conference on Prognostics and Health Management, IEEE PHM Conference, 2012, pp. 1-10.

[8] T. Sreenuch, A. Alghassi, S. Perinpanayagam, and Y. Xie; "Probabilistic Monte-Carlo Method for Modelling and Prediction of Electronics Component Life", (IJACSA) International Journal of Advanced Computer Science and Applications, Vol. 5, No. 1, 2014, pp. 96-104.

[9] S. Sarkar, X. Jin, and A. Ray, "Data-Driven Fault Detection in Aircraft Engines With Noisy Sensor Measurements", Journal of Engineering for Gas Turbines and Power, Vol. 133, ASME, August 2011, pp. 1-10.

[10] L. Jianhui, M. Madhavi, K. Pattipati, Q. Liu, M. Kawamooto, and S. Chigusa, "Model-based Prognostic Techniques Applied to Suspention System", IEEE Transactions on Systems, Man and Cybernetics, Part A: Systems and Humans, IEEE 2008, Vol. 38, Issue 5, pp. 1156-1168.

[11] P. Shetty, D. Mylaraswamy, and T. Ekambaram, "A hybrid prognostic model formulation system identification and health estimation of auxiliary power units", Aerospace Conference, IEEE, Big Sky, MT, USA, March 2006, pp. 1-10.

[12] A. K. Garga, K. T. McClintic, R. L. Campbell, Y. Chih-Chung, M. S. Lebold, T. A. Hay, and et al., "Hybrid reasoning for prognostic learning in CBM systems", Digital Object Identifier: IEEE Proceedings on Aerospace Conference, Big Sky, Montana, USA, vol.6, March 2001, pp 2957-2969.

[13] L. O. Chua , C. A. Desoer, and E. S. Kuh, "Linear and Nonlinear
Circuits", Mcgraw-Hill, 1st Ed. Edition, March 1987.

[14] D. Y. Gao, "Duality Principles in Nonconvex systems: Theory, Methods and Applications", Kluwer Academic Publishers, USA, ISBN 9781441948250, 2000

[15] G. E. Sharpe and, G. P. H. Styan, "Circuit Duality and the General Network Inverse", IEEE Transactions on Circuit Theory, Vol 12, IEEE 1965, pp. 22-27.

[16] J. Li, "Optimal Fault Diagnosis Based on Duality Principle for Linear Systems", Control and Decision IEEE Conference, July 2008, pp. 573577.

[17] R. W. Erickson, "Synthesis of switched-mode converters",IEEE Power Electron, PESC '83; Annual Power Electronics Specialists Conference, 14th, Albuquerque, NM, June 6-9, 1983, pp. 9-22.

[18] D. Maksimovic, "Synthesis of PWMand quasi-resonant DC-to-DC power converters," Ph.D. dissertation, California Inst. Technol., Pasadena, CA, USA, 1989.

[19] Y. S. Lee, "A systematic and unified approach to modeling switches in switch-mode power supplies," IEEE Trans. Ind. Electron., vol. IT-32, no. 4, Nov. 1985 , pp. 445-450.

[20] A. K. S. Bhat and F. D. Tan, "A unified approach to characterization of PWM and quasi-PWM switching converters: Topological constraints, classification, and synthesis," IEEE Trans. Power Electron., vol. 6, no. 4, Oct. 1991, pp. 719-726.

[21] M. Ogata and T. Nishi, "Topological criteria for switched mode dc-dc converters," in Proc. ISCAS, May 2003, vol. 3, pp. 184-187.

[22] D. H. Wolaver, "Basic constraints from graph theory for DC-to-DC conversion networks," IEEE Trans. Circuit Theory, vol. CT-19, no. 6, Nov. 1972, pp. 640-650.

[23] Y. Berkovich, A. Shenkman, A. Loinovici, and B. Axelrod, "Algebraic representation of DC-DC converters and symbolic method of their analysis," in Proc. IEEE 24th Convent. Elect. Electron. Eng., Eilat, Israel, 2006, pp. 47-51.

[24] M. Ogata and T. Nishi, "Topological conditions for passive switches in switching converters," in Proc. 18th IEEE ECCTD, 2007, pp. 898-901.

[25] T. Nishi, T. Oghishima, and M. Ogata, "Topological conditions on switched mode DC-DC converters," in Proc. ITC-CSCC, Jul. 2002, pp. 1129-1132.

[26] R. Severns, "Switch mode converter topologies make them work for you," Intersil, Inc., Milpitas, CA, USA, Appl. Bull. A035, 1980.

[27] B. W. Williams; "Generation and Analysis of Canonical Switching Cell DC-to-DC Converters". IEEE Transactions on Industrial Electronics, VOL. 61, NO. 1, January 2014, pp. 329-346.

[28] F. J. Rytkonen and R. Tymerski, "Modern Control Regulator Design for DC-DC Converters", Electrical and Computer Engineering Department Portland State University. [online]. Available from http://web.cecs.pdx. edu/ tymerski/ece451/Cuk_Control.pdf,2014.05. 14.

[29] A. Alghassi, S. Perinpanayagam, I. K. Jennions; "Prognostic capability evaluation of power electronic modules in transportation electrification and vehicle systems", IEEE, 15th IEEE Conference on Power Electronics and Applications (EPE), 2-6 Sept. 2013, 9 pages.

[30] A. Alghassi, S. Perinpanayagam, I. K. Jennions; "A simple state-based prognostic model for predicting remaining useful life of IGBT power module", IEEE, 15th European Conference on Power Electronics and Applications (EPE), 2013, 7 pages. 